\title{
IL RUOLO COGNITIVO DEI GESTI \\ NELL'EDUCAZIONE LINGUISTICA
}

\section{THE COGNITIVE ROLE OF GESTURES \\ IN LANGUAGE TEACHING}

\section{Moira De laco*}

\section{RIEPILOGO}

Questo contributo intende fornire argomenti utili per comprendere l'efficacia cognitiva dei gesti nella didattica delle lingue. Muovendo dall'influenza dei gesti su processi cognitivi quali i processi attentivi, la memoria, il problem solving e il ragionamento, si giungerà a mostrare le funzioni che i gesti possono svolgere nell'educazione linguistica. Il fine è quello di evidenziare l'esigenza per i docenti L2 di diventare sempre più consapevoli dell'uso strategico dei gesti nella didattica.

Parole chiave: gesti; attenzione congiunta; ${ }_{i}$ processi cognitivi, funzioni dei gesti; didattica delle lingue.

\section{ABSTRACT}

This paper aims to provide useful arguments to understand the cognitive role of gesture in language teaching. Moving from the influence of gesture on cognitive processes such as attention, memory, problem solving and reasoning, it will be shown the functions of gesture in language teaching in order to underline the necessity to make teachers of second language more aware of the use of gesture as strategy for teaching.

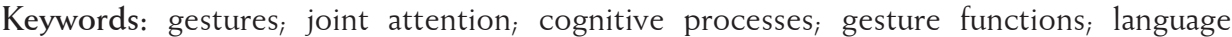
teaching.

\section{INTRODUZIONE}

Quando entrai la prima volta in una classe L2 presso il Goethe Institute di Roma non avevo mai studiato il tedesco e nessuna parola tedesca mi era familiare. Il docente entrò in classe, ci guardò e disse: "Guten Tag". Il piccolo gruppo di studenti davanti a lui restò in silenzio. Poi puntò il dito verso di me, muovendo il palmo della mano dal basso verso l'alto mi invitò ad alzarmi e con un ulteriore gesto (agitando la mano verso di sé), mentre diceva qualcosa che solo oggi sono in grado di distinguere come "kommen Sie", mi chiese di andare da lui alla lavagna. A quel punto, facendo il gesto dello scrivere, mi disse: "Schreiben Sie: Guten Tag". Intesi - anche grazie all'aiuto dei miei colleghi che, coinvolti con gesti d'animazione da parte del docente, si sforzarono insieme a me di capire - che dovevo scrivere sulla lavagna quanto da lui detto. Ricordo perfettamente che scrissi malamente quelle due parole che più di una volta il docente aveva ripetuto, per cui "Guten Tag" divenne "Cuten Tak". Usando gesti e parole il docente chiese ai miei colleghi di correggere quanto da me scritto scandendo ancora quelle

\footnotetext{
* Universidade de Bari, Itália. moiradeiaco@gmail.com

Orcid: https://orcid.org/0000-0001-5943-8267
} 
due parole: "Guten Tag". Molto di quello che in quel primo approccio alla lingua tedesca riuscì a capire fu frutto dell'azione integrata di parole e gesti usati con lo scopo di animare il gruppo classe, di informare circa quella forma di saluto e i suoi aspetti fonologici e di correggere gli errori di scrittura ${ }^{1}$.

In questo ricordo della prima esperienza come discente L2 è possibile rinvenire quanto Marion Tellier (2008) osserva a proposito dei gesti nella didattica delle lingue. Le funzioni dei gesti non si esauriscono certo alle prime fasi dell'educazione linguistica, ma attraversano quest'ultima costantemente modulandosi in base alle specifiche esigenze di ciascun livello di acquisizione della L2. Tellier classifica quelli che lei denomina "gesti pedagogici" distinguendoli in base a funzioni generali quali informare, animare e valutare. Per cui possiamo schematicamente dire che nei contesti di educazione linguistica si ricorre ai gesti con la funzione di informare gli studenti circa strutture grammaticali, lessicali o fonologiche rappresentando di volta in volta gli aspetti di cui si intende fornire conoscenza; si utilizzano poi i gesti di animazione per gestire il gruppo classe, ad esempio, assegnando i turni di parola o sollecitando la partecipazione degli studenti e si impiegano i gesti di valutazione per fornire feedback o evidenziare errori.

In questo contributo intendiamo evidenziare l'influenza esercitata dai gesti sui processi cognitivi, come l'attenzione e la memoria, con l'obiettivo di tracciare un'analisi del ruolo che i gesti ricoprono nella didattica delle lingue. Emergerà che gesti e parole sono un sistema integrato tanto della comunicazione quanto dell'educazione linguistica, per cui è essenziale rendere consapevole l'insegnante L2 del potenziale cognitivo dei gesti affinché egli possa adottarli proficuamente come strategia didattica.

\section{GESTI E LINGUA: UN SISTEMA INTEGRATO}

L'idea, argomentata da Tomasello (2008), che il linguaggio non possa essere considerato uno strumento innato della mente umana, ma sia piuttosto da intendere come il risultato di un adattamento biologico frutto dell'attività cooperativa umana, si basa sui suoi studi sull'uso dei gesti deittici e iconici da parte dei bambini. Tali studi hanno rivelato che lo sviluppo del linguaggio verbale e delle nostre strutture culturali è fondato sul carattere prettamente sociale e cooperativo dell'interazione umana. Alle origini delle nostre parole ci sono i gesti, forme espressive più primitive nel senso di più istintive e immediate, in grado di congiungere l'attenzione dei

1. Ricordo dell'autrice. 
parlanti permettendo il funzionamento della comunicazione. I gesti non si limitano a facilitare l'interazione nelle fasi di competenza linguistica elementare, bensì continuano a costituire la comunicazione umana anche a livelli più evoluti, assumendo un importante ruolo pragmatico nell'insegnamento. I gesti sono parte costitutiva del nostro modo di esprimerci: permettono di fare, oltre che parlare, vedere e sentire. Essi convivono con le parole, talvolta ci insegnano a usarle, delle volte ne prendendono il posto, altre ancora ne rafforzano i significati o facilitano la comprensione attraverso l'aggiunta di informazioni o la disambiguazione del discorso. La comunicazione umana si configura, dunque, come un sistema integrato di gesti e linguaggio verbale (CLARK, 1996; MCNEILL, 1998, 2000; KENDON, 2004). Ciò ha anche una spiegazione neurofisiologica: è stato dimostrato, infatti, che l'area di Broca deputata alla produzione e alla comprensione degli input verbali, si attiva anche quando siamo chiamati a comprendere i gesti (WILLEMS, ÖZYREK, e HAGOORT, 2007)

Nelle fasi di apprendimento di una lingua l'espressione gestuale risulta più diretta e trasparente di quella verbale. Occorre tenere presente che, secondo la Dual Coding Theory (CLARK, PAIVIO, 1991), la co-occorrenza di stimoli verbali e non verbali rende più efficace l'apprendimento. Da questo punto di vista si può osservare che l'uso dei gesti contestualmente alla presentazione del lessico favorisce l'apprendimento di quest'ultimo. Infatti, la doppia codifica degli input (PAIVIO, 1969), in modalità visiva oltre che uditiva, rende più efficace la comprensione e il recupero degli input verbali. Essa è stata testata nell'apprendimento delle lingue straniere mostrando effetti positivi (PAIVIO, DESROCHERS, 1980).

I gesti permettono di dirigere e concentrare l'attenzione nonché di rappresentare visivamente intenzioni, oggetti, aspetti e situazioni da concettualizzare (IANİ-FORMICHELLA, 2017). Essi svolgono un ruolo facilitante nell'apprendimento, precedono e predicono lo sviluppo del linguaggio verbale (TOMASELLO 2008) e sono in grado di influenzare positivamente l'apprendimento e la memorizzazione del lessico (DIADORI, 2013; IANİ, FORMICHELLA, 2017). A questo proposito lo studio del 2008 di Marion Tellier su un gruppo di bambini francofoni impegnato nell'apprendimento della lingua inglese ha rivelato gli effetti positivi, in termini di apprendimento, quando ai bambini venivano presentati gli input verbali in associazione a gesti e veniva successivamente chiesto loro di riprodurre l'input ricevuto insieme al gesto a esso associato. Questa associazione tra gesti e parole permette evidentemente di attivare anche la componente della memoria a breve termine responsabile dell'elaborazione del materiale visuo-spaziale, 
ovvero il taccuino visuo-spaziale, rafforzando l'elaborazione dell'input verbale a cui è deputato il ciclo fonologico.

La possibilità di codificare un concetto sia nella modalità visiva che in quella verbale, ne favorisce la memorizzazione e la rievocazione dell'input verbale risulta facilitata se associata al linguaggio gestuale. Ianì e Formichella (2017, p. 7) osservano, a questo proposito, che i gesti consentono di "mantenere vivide le immagini mentali nella componente visuo-spaziale alleggerendo il carico cognitivo della memoria di lavoro". Ci si potrebbe allora chiedere se i gesti - che sono in grado di innescare e supportare l'accesso e la rievocazione lessicale - giochino un ruolo attivo nella formazione dei concetti - che sarà poi a carico dei sistemi centrali e della memoria a lungo termine - o se si limitino a contribuire positivamente al recupero del lessico già archiviato.

\section{GESTI E PROCESSI COGNITIVI}

In accordo con alcune teorie dell'embodied cognition, possiamo dire che $\mathrm{i}$ gesti sono una parte costitutiva del nostro pensare e svolgono un ruolo nel processo di concettualizzazione. Pensiamo soprattutto, in tal senso, all'Information Packaging Hypothesis, secondo la quale i gesti sono coinvolti nella pianificazione concettuale del messaggio da verbalizzare (KITA, 2000, p. 163; ALIBALI, KITA e YOUNG, 2000 , p. 595) e rivestono una valenza cognitiva che comprende attività quali il ragionamento, il problem solving, l'apprendimento. L'Information Packaging Hypothesis rispetto alla Lexical Retrieval Hypothesis, che considera solo il ruolo facilitante dei gesti nel recupero del lessico, (KRAUSS, 1998; KRAUSS et al., 2000) sostiene che i gesti aiutino i parlanti "a esplorare, in modi di organizzazione alternativi, ciò che viene percepito e favoriscano la segmentazione di quest'ultimo in unità verbali" (ALIBALI, KITA E YOUNG, 2000, p. 610). Essi permettono dunque di "ri-rappresentare la conoscenza percettiva e motoria in forme verbali" (ALIBALI, KITA E YOUNG, 2000, p. 610) influenzando questa stessa rappresentazione attraverso la messa a fuoco di particolari aspetti di una situazione o di un elemento (ALIBALI, DI RUSSO, 1999; ALIBALI, KITA e YOUNG, 2000). In questo modo si ritiene che i gesti siano in grado di svolgere un ruolo determinante non solo per la concettualizzazione, ma anche per altre attività cognitive come i processi della memoria e l'attenzione.

L'uso dei gesti è strettamente correlato ai processi attentivi. Tomasello evidenzia come grazie alla deissi - sia nella sua funzione puramente deittica che in quella rappresentazionale in cui il referente non è presente nello spazio reale in cui il 
gesto viene praticato - l'intenzione del parlante viene compresa dal ricevente, per cui (in particolar modo nei contesti di apprendimento linguistico, ma non solo in essi) ha luogo quella che lui chiama "attenzione congiunta", grazie alla quale soltanto, come egli sostiene, "il linguaggio può svilupparsi e funzionare" (1995, pp. 105-106).

L'attenzione congiunta implica che ci siano almeno due persone che prestano attenzione comune a qualcosa o qualcuno o a una situazione nell'ambiente circostante e che siano consapevoli di questo comune prestare attenzione. Si tratta dunque di un fenomeno cognitivo di tipo sociale. Affinché la deissi funzioni, chi osserva il gesto deve assumere che chi lo compie sia un agente intenzionale con cui sta condividendo lo spazio visuale, le emozioni o i comportamenti. Ciò vale anche per chi compie il gesto: egli si aspetta che il suo osservatore sia in grado di condividere lo spazio visuale entro cui egli agisce, nonché di adottare il suo stesso focus attentivo e la sua stessa linea di comportamento (TOMASELLO, 1995, p. 112). Entro tale prospettiva, il gesto assume una funzione essenziale permettendo di organizzare la percezione e strutturare il pensiero e la comunicazione.

I processi attentivi non sono coinvolti solo nell'uso dei gesti deittici. Anche gli altri tipi di gesto - che avremo modo di riassumere nel prossimo paragrafo - richiedono uno sforzo attentivo. Attivando la modalità visiva, tutti i gesti implicano compiti di attenzione selettiva di tipo visivo, in quanto comportano la selezione della specifica parte dello spazio visivo in cui avviene il gesto e su cui l'attenzione è chiamata a concentrarsi. Ciascun gesto indirizza l'attenzione in base all'informazione da veicolare e configura un compito di attenzione focalizzata in cui è necessario concentrare l'attenzione sugli input pertinenti alla comunicazione. Se i gesti accompagnano le parole, inoltre, può essere necessario svolgere un compito di attenzione divisa in cui simultaneamente si presta attenzione a input diversi e in egual modo utili alla realizzazione dell'atto comunicativo.

\section{LE FUNZIONI DEI GESTI}

Le funzioni dei gesti sono differenti e non si limitano all'ambito comunicativo. Pensiamo, ad esempio, all'uso dei gesti in attività quali lo studio, la memorizzazione di una poesia, la preparazione di un talk. In casi come questi l'uso dei gesti si caratterizza come un supporto utile a visualizzare e chiarificare i concetti in modo da favorirne la memorizzazione e l'apprendimento. I gesti si configurano, dunque, come un'estensione della nostra attività cognitiva che assume, grazie a essi, un'organizzazione spaziale che facilita quella mentale. 
Sono state fornite diverse distinzioni dei gesti a seconda dei parametri di classificazione di volta in volta adottati. I gesti possono essere distinti in base al rapporto che intrattengono con il linguaggio verbale. Abbiamo quindi gli emblemi che sono i gesti indipendenti dalla comunicazione verbale. Tali gesti possono sostituirsi completamente alle espressioni verbali: pensiamo al gesto della mano alzata per fare stop. I gesti illustratori, invece, accompagnano le parole senza sostituirle. Essi hanno un ruolo adiuvante rispetto agli scopi della comunicazione e al linguaggio verbale.

Ci sono, poi, i gesti che esprimono gli stati emozionali, i cosiddetti indicatori emozionali. Non bisogna infatti dimenticare che la gestualità è in grado di veicolare le emozioni fornendo un'immagine dello stato emotivo in cui si trova il parlante. Il ricorso a questo genere di gesti risente di sostanziali differenze culturali, per cui ci sono culture in cui l'uso di indicatori emozionali è più diffuso di altre.

Infine, per completare il quadro della classificazione fornita da Eckman e Friesen (1969), dobbiamo menzionare i gesti regolatori, ovvero i gesti usati, ad esempio, per regolare i turni di parola nell'interazione linguistica, e i gesti adattatori che hanno lo scopo di riequilibrare gli stati di tensione visibili a livello somatico. L'uso dei gesti illustratori e regolatori è molto frequente e utile nei contesti di educazione linguistica.

I gesti illustratori, anche detti "gesti co-verbali" (TUMMILLO, 2016, p. 124), assumono un ruolo di rilievo nella didattica delle lingue e occorre che gli insegnanti L2 li utilizzino correttamente e con profitto in classe. Possiamo schematicamente dire di avere a che fare con le seguenti tipologie di gesti illustratori (MCNEILL, 1992; TUMMILLO, 2016; IANİ, FORMICHELLA, 2017):

1. i gesti deittici che permettono di indicare un oggetto, un luogo, una direzione a cui si fa riferimento (il dito puntato che accompagna "questo", "lì", "da quella parte" anche senza che vi sia un effettivo aggancio alla realtà, quindi anche in contesti immaginari o astratti);

2. i gesti iconici che rappresentano il contenuto semantico del discorso o il significato di una parola, in modo diretto e trasparente, riproducendolo analogamente nello spazio (ad esempio, i gesti delle dita corrispondenti ai numeri o il gesto che disegna una sfera in riferimento a oggetti come la palla);

3. i gesti metaforici attraverso i quali si rappresenta il contenuto di concetti astratti (ad esempio, le dita a forbice usate per invitare qualcuno ad accorciare un discorso) 
4. i gesti ritmici o motori, i quali sono denominati così in quanto seguono il ritmo del discorso riproducendone la prosodia attraverso forme gestuali standard indipendenti dai contenuti del discorso.

Gli emblemi, anche detti gesti simbolici, hanno carattere convenzionale e possono avere uno specifico valore culturale, ovvero possono assumere un significato diverso a seconda della cultura in cui si colloca il loro uso. Essi rivestono un interesse particolare nell'ambito dell'insegnamento delle lingue straniere proprio per via di questa specificità culturale. I gesti simbolici, infatti, sono in grado di sostituirsi alle espressioni verbali e richiedono una competenza interculturale da parte del discente per poter essere compresi (TUMMILLO, 2016, p. 214). È necessario che essi vengano appresi dal discente L2 e, pertanto, devono essere inclusi nel programma dei corsi di lingua straniera.

Diadori (2013, p. 13) ha evidenziato come lo sviluppo delle competenze pragmatiche in L2 renda necessario "l'uso condiviso di codici non verbali culturalmente specifici" come quello della gestualità. L'apprendimento dei gesti rientra nella competenza extralinguistica strettamente correlata alla competenza socio-pragmatica e interculturale che i discenti L2 devono acquisire (Balboni, 2015). Occorre assumere il ruolo specifico dei gesti convenzionali entro la cultura di riferimento della L2 giacché una mancata acquisizione delle competenze utili all'uso e alla comprensione di tali gesti può generare incomprensioni ed equivoci. Soprattutto per le lingue di area mediterranea, nelle quali la gestualità convenzionale svolge un ruolo essenziale nella comunicazione quotidiana, l'insegnamento della gestualità simbolica va considerato sin dai primi livelli di apprendimento della competenza linguistica, al fine appunto di evitare l'insorgere di fraintendimenti (DIADORI, 2013).

Nella prospettiva dell'uso della gestualità da parte del discente L2, un'ulteriore distinzione di cui rendere conto e proposta negli studi più recenti dedicati alla gestualità nell'apprendimento della L2, è quella tra gesti rappresentazionali e gesti pragmatici (GRAZIANO, GULLBERG, 2013). I gesti rappresentazionali, anche detti gesti referenziali, sono quelli che esprimono un contenuto semantico riferendosi a proprietà referenziali quali, ad esempio, la forma o la misura di qualcosa o qualcuno. I gesti che abbiamo classificato come illustratori possono svolgere la funzione di gesti rappresentazionali. I gesti pragmatici, invece, non esprimono alcun contenuto referenziale e ritraggono elementi del discorso o commentano la produzione linguistica del parlante segnalando, per esempio, eventuali interruzioni o incertezze che la caratterizzano (KENDON, 2004). A questo proposito uno studio ha mostrato come in casi di disfluenza i parlanti ricorrano spesso ai gesti pragmatici 
che commentano le difficoltà d'interazione dovute alla disfluenza (GRAZIANO, GULLBERG, 2013). Lo stesso studio ha anche evidenziato come i discenti adulti L2 siano soliti ricorrere ai gesti rappresentazionali per indurre l'interlocutore a fornire loro supporto per il lessico (GRAZIANO, GULLBERG, 2013). Questi elementi rivelano che gesti e parole costituiscono un sistema integrato non solo dal punto di vista della comprensione, ma anche per quello della produzione linguistica. E ciò vale tanto nelle situazioni comunicative, quanto in contesti didattici.

\section{L'USO DEI GESTI PER L'EDUCAZIONE LINGUISTICA}

Esiste un uso dei gesti che assolve funzioni cognitive e pragmatiche essenziali alla didattica come:

1. agevolare la comprensione e la memorizzazione del lessico attraverso gesti iconici e deittici associati al linguaggio verbale con funzione rappresentazionale e di informazione;

2. trarre una risposta dagli studenti mediante il ricorso a gesti illustratori con funzione di animazione del gruppo classe;

3. fornire riscontri correttivi rappresentando tramite $\mathrm{i}$ gesti la risposta corretta e facendo così leva sull'efficacia dello sfruttamento di una modalità rappresentazionale alternativa a quella verbale in cui era stata formulata la risposta errata da parte dello studente (TALEGHANI-NIKAZM，2008; DIADORI, 2013).

Ricorrendo ai gesti co-verbali l'insegnante L2 è in grado di dirigere l'attenzione modificando a livello pragmatico la ricezione dell'input verbale. Inoltre, l'uso dei gesti da parte del docente comporta un effetto mirroring per il quale gli allievi si ritrovano a riprodurre inconsapevolmente gli stessi gesti usati dall'insegnante: si tratta, come Diadori scrive, di un "atteggiamento adattivo e imitativo tipico del ruolo dell'allievo nella classe di lingua" (2013, p. 3).

Ogni tipologia gestuale fra quelle descritte nel precedente paragrafo è in grado di svolgere una specifica funzione didattica nella classe di lingua e, pertanto, la funzione didattica dei gesti merita di essere analizzata e assunta nella sua importanza.

I gesti deittici permettono quella che con Tomasello possiamo chiamare "attenzione congiunta". Si tratta della possibilità di richiamare l'attenzione dell'altro dirigendola su quanto vogliamo comunicargli e dunque di generare comprensione 
e unione di intenti. Questo tipo di gesti è fondamentale nelle prime fasi di apprendimento linguistico dove il riferimento immediato, trasparente, al referente è spesso costitutivo per l'insegnamento del lessico e per la comunicazione da parte del discente, il quale si trova a ricorrere alla deissi come supporto sostitutivo dell'espressione verbale che ancora deve apprendere.

I gesti iconici possono fornire un valido supporto sostitutivo dell'espressione verbale da apprendere oppure possono essere usati per introdurre il lessico agevolandone la comprensione e la memorizzazione.

I gesti metaforici possono apparire meno immediati rispetto alle precedenti due tipologie gestuali: in realtà, il loro grado di metaforicità - una caratteristica pervasiva nel nostro sistema concettuale (LAKOFF, JOHNSON, 1980) - espresso su un canale visivo immediato piuttosto che su quello verbale, li rende strumenti didattici indubbiamente utili: attraverso i gesti metaforici è possibile tradurre metafore concettuali altamente utilizzate per renderne più efficace l'insegnamento o per rendere gli studenti consapevoli riguardo determinati aspetti linguistici. Pensiamo, in tal senso, all'esempio proposto da Tummillo del "gesto metaforico del contenente" in cui un locutore ponendo le mani in posizione parallela tiene tra di esse, in modo astratto, un oggetto immaginario. Un gesto astratto può essere utilizzato per rendere lo studente consapevole della struttura sillabica delle parole (2016, pp. 125, 129).

I gesti ritmici possono rappresentare la prosodia e si mostrano particolarmente utili per l'apprendimento della giusta accentazione.

I gesti convenzionali o emblemi, come abbiamo già avuto modo di evidenziare, non rientrano fra i gesti a cui ricorrere come strategia didattica. Essi devono, invece, essere insegnati e appresi per via della loro valenza culturale.

\section{CONCLUSIONI E PROSPETTIVE FUTURE}

Abbiamo cercato di evidenziare la rilevanza del ruolo dei gesti nell'educazione linguistica, sin dal primo approccio con una L2, sottolineando gli aspetti cognitivi implicati nell'uso della gestualità in contesti didattici. Riteniamo sia necessario continuare a riflettere sulle funzioni dei gesti nella didattica tenendo conto proprio della loro portata cognitiva. Diversi studi hanno dimostrato come l'uso dei gesti da parte dell'insegnante L2, ma anche dell'apprendente di lingua renda più efficace l'apprendimento agevolando la comprensione e la memorizzazione degli input verbali (GOLDIN-MEADOW, 2004, p. 319; IANÌ, FORMICHELLA, 2017). Goldin-Meadow $(2003,2004)$ si è concentrata anche sull'uso dei gesti da parte 
dei bambini in contesto didattico sottolineando come tali gesti siano in grado di rivelare all'insegnante lo stato delle conoscenze del bambino $\square$ soprattutto di quelle implicite che il bambino non è ancora in grado di esprimere verbalmente tracciando la direzione dell'intervento didattico da intraprendere. Determinante da questo punto di vista risulta l'accordo e il disaccordo tra il gesto e l'espressione verbale: un bambino che dice sette e con le mani compie il gesto "sei" o che calcola quattro più tre con le dita, ma dice sei come risultato, suggerisce all'insegnante qualcosa di diverso circa lo stato del suo apprendimento rispetto a quanto suggerisca un bambino che correttamente dice sette quando calcola con i gesti quattro più tre e in corrispondenza dell'espressione sette compie il gesto sette.

La direzione da seguire per quanto concerne la didattica delle lingue è quella di un uso sempre più consapevole dei gesti al fine di sfruttare tutto il loro potenziale cognitivo in termini di efficientamento del sistema attentivo e della memoria di lavoro. La capacità dei gesti rendere più efficienti l'attenzione e la memoria agevola l'apprendimento linguistico. Occorre, dunque, ampliare l'analisi della capacità didattica di ciascuna tipologia gestuale entro il quadro delle tre funzioni pedagogiche di informazione, animazione e correzione individuate da Tellier (2006; 2008), in modo da implementare l'uso strategico dei gesti nella didattica. Nella prospettiva della didattica delle lingue non va inoltre sottovalutato il ruolo giocato dall'uso dei gesti da parte del discente. Esso costituisce per il docente un indice importante, anche dal punto di vista della componente affettivo-emotiva, per verificare l'apprendimento e per indirizzare le successive azioni didattiche. Da questo punto di vista, pensiamo che un'indagine sul possibile ruolo dei cosiddetti indicatori emozionali, con le loro specificità culturali, possa fornire esiti interessanti.

\section{RIFERIMENTI BIBLIOGRAFICI}

ALIBALI, M.W., DIRUSSO, A.A. (1999). The function of gesture in learning to count: More than keeping track. Cognitive Development. v. 14, pp. 37-56.

ALIBALI, M. W., KITA, S., YOUNG, A. J. (2000). Gesture and the Process of Speech Production: We Think, Therefore We Gesture. Language and Cognitive Process. v. 15, n. 6, pp. 593-613.

BALBONI, P. E. (2015). Le sfide di Babele. Insegnare le lingue nelle società complesse. Milano: Utet, $4^{\mathrm{a}}$ Ed. 2020. 
DIADORI, P. (2013). Gestualità e didattica della lingua straniera: questioni interculturali. In: Borello P., Luise M.C. (a cura di), Aspetti comunicativi e interculturali nell'insegnamento delle lingue. Alessandria: Edizioni dell'Orso. Disponibile online: http://www.siena-art. com/diadori/Testi/08iGESTI.pdf.

CLARK, H. (1996). Using Language. Cambridge: Cambridge University Press.

EKMAN, P., FRIESEN, W. V. (1969). The repertoire of nonverbal behavior: Categories, origins, usage, and coding. Semiotica. n. 1, pp. 49-98.

GOLDIN-MEADOW, S. (2003). Hearing Gesture: How Our Hands Help Us to Think Cambridge (Massachusetts): The Belknap Press of Harvard University Press.

GOLDIN-MEADOW, S. (2004). Gesture's Role in the Learning Process. Theory into Practice, v. 43, n. 4, pp. 314-321.

GRAZIANO, M., GULLBERG, M. (2013). Gesture production and speech fluency in competent speakers and language learners. In: Proceedings of the Tilburg Gesture Research Meeting (TiGeR). Tilburg: Tilburg University.

IANİ, F., FORMICHELLA, M. (2017). Il ruolo cognitivo dei gesti. Giornale Italiano di Psicologia. n. 4, pp. 849-876. Disponibile online: https://iris.unito.it/handle/2318/1659812.

KENDON, A. (2004). Gesture. Visible action as utterance. Cambridge: Cambridge University Press.

KITA, S. (2000). How representational gestures help speaking. In: McNeill D. (Ed.), Language and gesture: Window into thought and action, Cambridge, UK: Cambridge University Press pp. $162-185$.

KRAUSS, R.M. (1998). Why do we gesture when we speak? Current Directionsin Psychological Science. v. 7, pp. 54-60.

KRAUSS, R. M. et al. (2000). Lexical gestures and lexical access: A process model. In: McNeill D. (Ed.), Language and gesture. Cambridge: Cambridge University Press, Cambridge, pp. 162-185.

LAKOFF, G., JOHNSON, L. (1980). Metaphors We Live by, Chicago: Chicago University Press. Trad. Metafora e vita quotidiana. Milano: Bompiani, 1998.

MCNEILL, D. (1992). Hand and Mind. What Gestures Reveal about Thought. Chicago (Illinois): University of Chicago Press. 
MCNEILL, D. (1998). Speech and gesture integration. In: Iverson, J. M., Goldin-Meadow, S. (Eds.). The nature and functions of gesture in children's communication. San Francisco: Jossey-Bass, pp. 11-27.

MCNEILL, D. (Ed.) (2000). Language and gesture: Window into thought and action. Cambridge, UK: Cambridge University Press.

PAIVIO, A. (1969). Mental imagery in associative learning and memory. Psychological Review. v. 76, n. 3, pp. $241-263$.

PAIVIO, A., DESROCHERS, A. (1980). A dual-coding approach to bilingual memory. Canadian Journal of Psychology. v. 34, pp. 390-401.

TALEGHANI-NIKAZM, C. (2008). Gestures in Foreign Language Classroom: An Empirical Analysis of their Organization and Function. In: Bowles, M. (Ed.). Selected Proceedings of the 2007 Second Language Research Forum. Somerville, MA: Cascadilla Proceeding Project, pp. 229-238.

TELLIER, M. (2006). L'impact du geste pédagogique sur l'enseignement-apprentissage des langues étrangères: Étude sur des enfants de 5 ans. Tesi di dottorato non pubblicata. Université Paris 7 - Denis Diderot, Paris.

TELLIER, M. (2008). The Effect of Gestures on Second Language Memorisation by Young Children. Gesture. v. 8, n. 2, pp. 219-235.

TOMASELLO, M. (1995). Joint attention as social cognition. In: Dunham, P. Moore, C. (Eds.), Joint attention. Its Origins and Role in Development. Hillsdale, NJ: LEA, pp. 103-129.

TOMASELLO, M. (2008). Origins of Human Communication, Massachusetts Institute of Technology. Trad. Le origini della comunicazione umana. Milano: Raffaello Cortina Editore, 2009.

TUMMILlO, F. (2016). Gestualità e didattica L2: spunti teorici e piste di lavoro. Lend. n. 2, pp. $122-130$.

WILLEMS, R. et al. (2007). When language meets action: The neural integration of gesture and speech. Cerebral Cortex. v. 17, pp. 2322-33.

Recebido: 19/7/2019

Aceito: $23 / 6 / 2020$

Publicado: 3/8/2020 